\title{
Viscoelastic Behavior of Poly(methyl methacrylate) Networks with Different Cross-Linking Degrees
}

\author{
N. M. Alves, ${ }^{\dagger,}$ J . L. Gómez Ribelles, ${ }^{\S}$ J . A. Gómez Tejedor, ${ }^{\perp}$ and J . F. Mano*,t, \\ Polymer Engineering Department, University of Minho, Campus of Azurém, \\ 4800-058 Guimarães, Portugal; 3B's Research Group-Biomaterials, Biodegradables and \\ Biomimetics, University of Minho, 4710-057 Braga, Portugal; Center for Biomaterials and \\ Department of Applied Thermodynamics, Universidad Politécnica de Valencia, P.O. Box 22012, \\ E-46071 Valencia, Spain; and Center for Biomaterials and Department of Applied Physics, \\ Universidad Politécnica de Valencia, Escuela Universitaria de Informática, E-46022 Valencia, Spain
}

Received October 29, 2003; Revised Manuscript Received March 4, 2004

\begin{abstract}
The influence of the cross-linking degree on the dynamics of the segmental motions close to the glass transition of poly(methyl methacrylate), PMMA, networks was investigated by three different mechanical spectroscopy techniques: thermally stimulated recovery (TSR), dynamic mechanical analysis (DMA), and creep. The application of the time-temperature superposition principle to isothermal DMA and creep results permitted to successfully construct master curves for PMMA networks with distinct cross-linking degrees. The former results were fitted to the KWW equation. The obtained variation of $\beta_{\mathrm{KWW}}$ for the distinct networks indicated that the relaxation curves tend to broaden as the cross-linking degree increases. TSR results clearly revealed a significant shift of the $\alpha$-relaxation to longer times and a broader relaxation as the cross-linking degree increases, what was also observed by DMA and creep. A change from a Vogel to an Arrhenius behavior was detected by the three techniques with the decrease of temperature bel ow $\mathrm{T}_{\mathrm{g}}$. The temperature dependence of the apparent activation energies $\left(\mathrm{E}_{\mathrm{a}}\right)$ was calculated from DMA, creep, and TSR experiments; above $T_{g}$ a good agreement was seen between the $E_{a}$ values for all the techniques. Furthermore, the effect of the cross-linking degree on the fragility of PMMA networks was evaluated. For these materials an increase of fragility with increasing cross-linking degree was observed.
\end{abstract}

\section{Introduction}

Intermolecular constraints play an important role in the segmental dynamics of all polymeric systems in the bulk state. ${ }^{1,2} \mathrm{~A}$ particular case in this context is that of cross-linked polymers. Several works have studied the influence of the cross-linking degree on the $\alpha$-relaxation by distinct techniques, such as dielectric spectroscopy, dynamic mechanical analysis, or creep and stressstrain measurements. ${ }^{3-9}$ Those studies revealed that the most striking effects caused by the increase of crosslinker concentration is the broadening of the relaxation and the slowing down of the segmental dynamics, which implies a shift of the transition zone to longer times or lower frequencies. In addition, the KWW asymmetry can be removed as seen, for example, by dielectric spectroscopy. ${ }^{5}$

Several molecular models have been devel oped since the last half of the 20th century to describe the behavior of polymer networks, all based on the microscopic picture of entropic elasticity of chains, and were recently reviewed. ${ }^{10}$ Basically they can be divided into unconstrained network models such as the affine model 11,12 or the phantom model ${ }^{13,14}$ and constrained network models based on the reptation concept, a term first introduced by de Gennes ${ }^{15}$ and developed by Doi and

\footnotetext{
† Polymer Engineering Department, University of Minho.

\# 3B's Research Group-Biomaterials, Biodegradables and Biomimetics, University of Minho.

$\S$ Center for Biomaterials and Department of Applied Thermodynamics, Universidad Politécnica de Valencia.

$\perp$ Center for Biomaterials and Department of Applied Physics, Universidad Politécnica de Valencia.

* Corresponding author: Tel +351-253510320; fax +351253510339; e-mail jmano@dep.uminho.pt.
}

Edwards. ${ }^{16,17}$ In the affine model it is assumed that the cross-link points in the network are fixed at their mean positions and deform affinely. In the phantom model the individual chains and cross-link points may pass through one another as if they had no material existence, and fluctuations of these points about their mean positions are allowed and unaffected by the state of strain in the network. In the constrained models it is considered that, due to the presence of cross-links, large-scale motions occur by reptation, which means that they relax by diffusion along their own contours among topological obstacles coming from neighboring chains; for length scales shorter than the average distance between entanglements the dynamics proceeds through normal modes as in nonentangled polymers.

The behavior of non-cross-linked PMMA in the glass transition region has been extensively studied by distinct techniques (see for example refs 18 and 19), which makes this polymer a suitable system to investigate and improve the understanding of the cross-linking effect on this relaxation. Moreover, in the past years PMMA has been largely utilized as a component of interpenetrating polymer networks (IPNs). ${ }^{20,21}$ The phase morphol ogy of IPN s strongly depends, among other factors, on the cross-linking density of the system because this is a competitive process between the natural tendency of phase separation and its impossibility due to network formation and consequent appearance of chemical crosslinks. As a consequence, the physical and mechanical properties of a given IPN can be modified by varying (among other factors) the cross-linking density, and this is precisely the main reason for the growing interest in this kind of material. In this context, the study of the influence of this factor on the viscoelastic properties of 
PMMA can be relevant for a better understanding of the role of cross-links on the segmental mobility of a given IPN where PMMA is a component.

The aim of the present work is to establish the influence of the cross-linking degree on the viscoelastic properties of PMMA in the region of the $\alpha$-relaxation, in which the mechanical response is due to segmental dynamics, while larger scale motions can be influenced by reptation processes. ${ }^{7}$ This study includes the employment of different but complementary mechanical spectroscopy techniques and, in particular, the use of the nonconventional technique known as thermally stimulated recovery (TSR).

The advantages of using thermally stimulated methods are rel ated to the access of low equivalent frequencies, of about $10^{-3}-10^{-2} \mathrm{~Hz}^{22}$ associated with the possibility of resolving complex transitions into narrow distributions of characteristic times. This capability is achieved with the thermal sampling (TS) procedure that will be described in the Experimental Section. Also, its high sensitivity permits to investigate the glass transition behavior not only for amorphous systems but also for semicrystalline or cross-linked polymers where the glass transition is not so pronounced. The segmental dynamic behavior of PMMA was already studied by thermally stimulated methods, essentially by thermally stimulated currents, ${ }^{23-25}$ but some works of thermally stimulated creep $26-28$ can be found. However, to our knowledge, this is the first time that the study of the effect of cross-linking degree on the $\alpha$ relaxation of PMMA by TSR is performed.

In addition, dynamic mechanical analysis (DMA) and creep were also used to characterize the viscoelastic behavior of PMMA networks. The use of several techniques, each of them stressing different aspects and giving distinct details of the observed relaxation process, in this case the $\alpha$ relaxation, allows to have a more complete picture of the mechanisms associated with segmental mobility.

It is worth mentioning that independently of the technique used and the structure of the glass-forming material (that may include low molecular or polymeric systems) the $\alpha$-relaxation exhibits general features, namely (i) the non-Debye behavior, where the KWW model is currently used for describing the shape of the relaxation curves, 29,30 and (ii) the non-Arrhenius temperature dependence of the characteristic relaxation times often described by the Vogel-Fulcher-TammanHesse (VFTH) law. ${ }^{31-33}$

The effect of cross-linking on the nonexponential and non-Arrehnius behavior of the $\alpha$-relaxation is investigated in this work. In particular, the fragility concept, popularised by Angell, ${ }^{34}$ was applied in order to compare the temperature dependence of the characteristic times for samples with distinct cross-linking densities.

\section{Experimental Section}

2.1. Materials. The materials studied were cross-linked poly(methyl methacrylates), PMMA, synthesized by free radical addition polymerization of methyl methacrylate (Aldrich, $99 \%$ pure) using as photoinitiator 0.13 wt \% of benzoin (Scharlau, 98\% pure). The samples were synthesized between two glass plates to form sheets of approximately $0.5 \mathrm{~mm}$ thick. Samples with distinct cross-linking degrees were prepared by adding $0.5,1,5,8,9$, and 10 wt $\%$ of ethylene glycol dimethacrylate, EGDMA (Aldrich, 98\% pure). The cross-links prevent permanent flow above the glass transition temperature $T_{g}$, allowing to perform both the DMA and TSR experi- ments across the glass transition. The monomer, cross-linking agent, and initiator were used as received without further purification. Polymerization took place at room temperature for $24 \mathrm{~h}$ under UV radiation. The low molecular weight substances remaining in the samples after polymerization were extracted with ethanol for $24 \mathrm{~h}$ and then dried in vacuo at $70^{\circ} \mathrm{C}$ for several weeks until the weight remains constant. Finally, the samples were dried in vacuo at $180{ }^{\circ} \mathrm{C}$ for $1 \mathrm{~h}$ in order to eliminate possible residues that still remain in the sample. The samples are referred in the text as PMMA0.5, PMMA1, ..., where the number after the sample refers to the amount of cross-linking agent.

2.2. DMA Experiments. The dynamic mechanical analysis (DMA) experiments were performed in a Seiko DMS210 analyzer at both isothermal and nonisothermal conditions for all the samples in the extension mode. Samples with rectangular geometry and dimensions $14 \times 4.4 \times 0.5 \mathrm{~mm}$ were used.

The nonisothermal runs were carried out at $1^{\circ} \mathrm{C} / \mathrm{min}$, from room temperature to $200{ }^{\circ} \mathrm{C}$ at $1 \mathrm{~Hz}$. The aim of these runs was to locate the glass transition region for each sample.

The isothermal experiments were carried out in the glass transition region of the samples: between $\sim 50$ and $\sim 160{ }^{\circ} \mathrm{C}$ for PMMA0.5 and PMMA1 and between $\sim 50$ and $\sim 170{ }^{\circ} \mathrm{C}$ for PMMA5 and PMMA9, every $2{ }^{\circ} \mathrm{C}$. At each temperature the frequency was scanned from 0.01 to $20 \mathrm{~Hz}$.

2.3. Creep Experiments. Creep experiments were performed in a SeikoTMA/6000 equipment in the extension mode. Samples with rectangular geometry and dimensions $20 \times 4 \times$ $0.5 \mathrm{~mm}$ were used. The maximum deformation of the sample was $1 \%$. The sample length was measured as a function of time at equal time intervals in logarithmic scale, and then, the creep compliance $\mathrm{D}(\mathrm{t})$ was calculated. After creep, the stress was removed, and the sample was recovered for a time twice the creep time. The experiment has been carried out at different temperatures, from 108.3 to $153.6^{\circ} \mathrm{C}$ every $4.5^{\circ} \mathrm{C}$ for PMMA1, from 122.9 to $150.6^{\circ} \mathrm{C}$ every $0.5^{\circ} \mathrm{C}$ for PMMA5, from 128.6 to $166.4{ }^{\circ} \mathrm{C}$ every $4.7^{\circ} \mathrm{C}$ for PMMA8, and from 134.6 to $167.2^{\circ} \mathrm{C}$ every $4.7^{\circ} \mathrm{C}$ for PMMA10.

2.4. TSR Experiments. The thermally stimulated recovery (TSR) experiments were carried out in a DMA7e Perkin-EImer analyzer (with a controlled cool ing accessory) in the extension mode. In this case just the PMMA0.5, PMMA5, and PMMA9 samples were analyzed. These samples had a rectangular geometry and dimensions $8 \times 1 \times 0.5 \mathrm{~mm}$. Continuous flux of high-purity helium (flow rate of $\sim 28 \mathrm{~cm}^{3} \mathrm{~min}^{-1}$ ) was used to improve heat transfer throughout the sample surroundings during the experiments.

In a "global" TSR experiment a static stress is imposed to the sample at a sufficient high temperature $\left(\mathrm{T}_{\sigma}\right)$ in order that in the resulting strain all molecular processes are involved; i.e., all characteristic times, $\tau\left(\mathrm{T}_{\sigma}\right)$, are small enough to quickly respond to the mechanical stimulus. During this creep process the sample is cool ed to a lower temperature $\left(T_{0}\right)$, at which the characteristic times are so high that any recovery process could not occur at a reasonable time scale. During a controllable heating process those times are progressively reduced, and when they reach sufficiently small values, the recovery process may be observed as sudden decreases of the $\epsilon(T)$ line (or peaks in a $\mathrm{d} \epsilon / \mathrm{dT}$ plot). The different translational/rotational mobility of the material may be then investigated by observing the $\mathrm{d} \epsilon /$ dT peaks in the temperature axis.

In this work the thermal sampling (or windowing), TS, procedure was followed to experimentally decompose the complex $\alpha$-relaxation process: a static stress, $\sigma_{0}$, is applied during an isothermal period, $\mathrm{t}_{\sigma}$, at a creep temperature $\mathrm{T}_{\sigma}\left(\mathrm{T}_{\sigma}\right.$ varies from experiment to experiment in the $\alpha$-relaxation region of each sample) and during a temperature program at constant rate between $\mathrm{T}_{\sigma}$ and $\mathrm{T}_{\sigma}-\Delta \mathrm{T}_{\mathrm{w}}$. Then without any stress the strain is partially recovered during an isothermal period at $\mathrm{T}_{\sigma}-\Delta \mathrm{T}_{\mathrm{w}}$ followed by a cooling to $\mathrm{T}_{0}\left(\mathrm{~T}_{0} \ll \mathrm{T}_{\sigma}\right)$. Finally, the strain is measured, as a function of temperature, during a heating at constant rate $(\beta=\mathrm{dT} / \mathrm{dt})$ up to a temperature well above $\mathrm{T}_{\sigma}$. In all the experiments carried out in this work we used $\beta=4{ }^{\circ} \mathrm{C} / \mathrm{min}, \Delta \mathrm{T}_{\mathrm{w}}=3^{\circ} \mathrm{C}, \mathrm{T}_{0}=\mathrm{T}_{\sigma}-50$ ${ }^{\circ} \mathrm{C}, \mathrm{t}_{\sigma}=4 \mathrm{~min}$, and $\mathrm{t}_{\mathrm{r}}=4 \mathrm{~min}$. It was assumed that the applied 


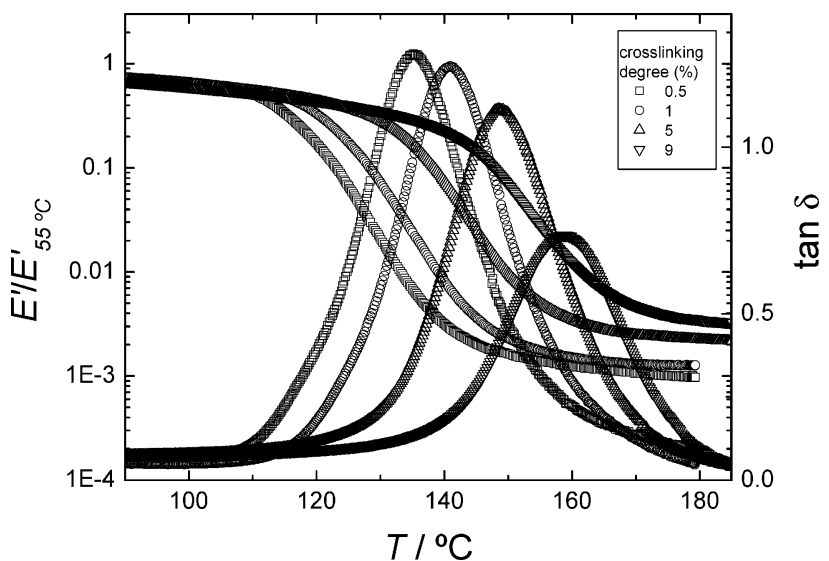

Figure 1. Storage modulus $\left(\mathrm{E}^{\prime}\right)$ and loss factor $(\tan \delta)$ against temperature for PMMA with different cross-linking degrees. The runs were carried out at $1^{\circ} \mathrm{C} \mathrm{min}^{-1}$ and for a frequency of $1 \mathrm{~Hz}$.

$\sigma_{0}$ values (different for the distinct experiments) were sufficiently small to ensure the studies in the linear viscoelastic regime, as the resulting maximum strain was always lower than $1.5 \%$. More details about this technique can be found elsewhere. ${ }^{35,36}$

\section{Results and Discussion}

3.1. DMA and Creep Data. The isochronal DMA scans of the analyzed PMMA networks in the $\alpha$-relaxation region are presented in Figure 1 as a function of the amount of EGDMA. A shift to higher temperatures of the maximum of the loss factor $(\tan \delta)$ is observed as the cross-linking density increases: 135.2, 141.0, 149.3, and $159.2{ }^{\circ} \mathrm{C}$ for PMMA containing EGDMA in proportions $0.5,1,5$, and $9 \%$, respectively. These changes on the dynamic mechanical spectrum have been al ready observed for PMMA samples cross-linked with the same agent. ${ }^{20}$ Note that for these samples $\tan \delta$ was chosen to represent the loss behavior in Figures 1 and 2 because, as typically observed in PMMA samples, ${ }^{37}$ the $\mathrm{E}^{\prime \prime}$ curves do not present a well-defined maximum due to the superposition between the $\alpha$ and the $\beta$ relaxations. The increase of the apparent glass transition temperature can be explained by an increase in the chain connectivity that leads to a decrease of the free volume and, hence, to the slowing down of the dynamics of the relaxation as the cross-linker concentration increases. Also, as the cross-linking degree increases, the relative amount of free linear polymeric segments and consequently the $\tan \delta$ peak intensity decrease. The elastic modulus $\mathrm{E}^{\prime}$ remains constant in the vitreous region, but an increase of $E^{\prime}$ in the elastomeric plateau is observed as the cross-linking degree increases. The cross-linking degree does not affect the viscoelastic properties of polymers in the glassy state as has been proposed elsewhere. ${ }^{8}$

The number of monomeric units between cross-links, $v$, for the PMMA samples was calculated both from stoichiometry $\left(v_{\mathrm{st}}\right)$ and from the elastomeric plateau modulus ( $v_{\text {exp }}$ ) because in this region the behavior of the material is mainly explained in terms of entropic elasticity, according to the theory of rubber elasticity: ${ }^{38}$

$$
v_{\exp }=\frac{3 \mathrm{RT} \rho \mathrm{A}_{\phi}}{\mathrm{M}_{\mathrm{m}} \mathrm{E}^{\prime}}
$$

where $\rho$ is the density in the elastomeric region, $\mathrm{R}$ is
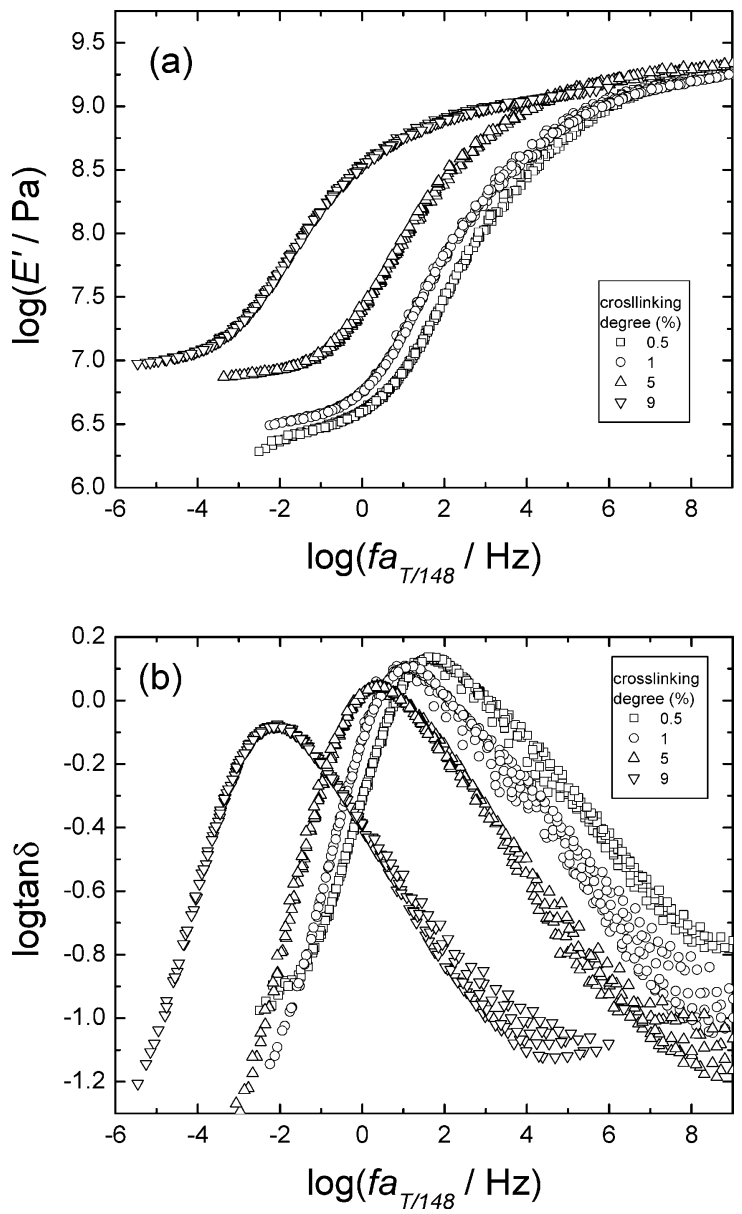

Figure 2. (a) Storage modulus ( $\left.E^{\prime}\right)$ and (b) loss factor (tan $\delta$ ) master curves at a reference temperature of $148^{\circ} \mathrm{C}$ for PMMA with different cross-linking degrees.

the ideal gas constant, $\mathrm{M}_{\mathrm{m}}$ is the molecular weight of the monomeric unit, $\mathrm{T}$ is the absolute temperature, $\mathrm{E}^{\prime}$ is the modulus in the elastomeric region, and $A_{\phi}$ is a factor that depends on the functionality of the crosslinking agent (for EGDMA the functionality is 4).

This equation assumes that the network is perfect in the sense that all chains in the network are effective in giving rise to the elastic stress. However, a number of network imperfections are possibl $\mathrm{e}^{39}$ like, for example, physical entangl ements, terminal chains, or intrachain loops.

The rubber elasticity theory ${ }^{39}$ can be based on the assumption that the cross-links behave according to a fully mobile-phantom model or, instead, according to an immobile-affine model. Of course, real networks exhibit a behavior that is between these two extreme situations; i.e., not all cross-links deform affinely but, on the other hand, steric interactions are strong enough to prevent the full mobility. The real situation is difficult to quantify. In this work the number of monomeric units between cross-links ( $v_{\text {exp }}$ ) was calculated at the rubbery plateau as a function of the cross-linking degree using the affine model, where $A_{\phi}=1$, to compare these values with the corresponding stoichiometric values $\left(v_{\text {st }}\right)$. Values of $E^{\prime}$ in the elastomeric region were obtained from Figure 1, and values of $\rho$ for PMMA as a function of temperature were listed in ref 40 . The obtained results are in Table 1.

For contents of cross-linker up to 5\% of EGDMA it can be seen that the stoichiometric values are higher than those calculated with eq 1, which can be explained, 
Table 1. Monomeric Units between Cross-Link Points Obtained from the Experimental Modulus $\left(v_{\exp }\right)$ through Rubber Elasticity Theory and from a Stoichiometric Calculation ( $v_{\mathrm{st}}$ ) for PMMA with Different Cross-Linking Degrees

\begin{tabular}{ccc}
\hline $\begin{array}{c}\text { cross-linking } \\
\text { degree (\%) }\end{array}$ & $v_{\exp }$ & \multicolumn{1}{c}{$v_{\text {st }}$} \\
\hline 0.5 & 34.7 & 199.5 \\
1 & 24.2 & 98.0 \\
5 & 14.3 & 18.8 \\
9 & 11.8 & 10.0
\end{tabular}

for example, by the appearance of physical entanglements. F or the highest content of cross-linker (9\%) the inverse situation is observed; i.e., the number of elastically active chains is lower than the value predicted by stoichiometry. This means that factors favorable to the increase of $\mathrm{E}^{\prime}$ (such as physical entanglements) are predominant than factors that reduce $E^{\prime}$ (for example, chains with a free extreme).

From the isothermal scans in the glass transition region the master curves for storage modulus $\left(E^{\prime}\right)$ and loss factor $(\tan \delta)$ were obtained as a function of reduced frequency $\mathrm{fa}_{\mathrm{T}}$ and for a reference temperature $\mathrm{T}_{\text {ref }}=148$ ${ }^{\circ} \mathrm{C}$ (Figure 2). The master curves were constructed by simple shifting of the isothermal results al ong the $\log f$ scale, according to the time-temperature superposition principle. ${ }^{41} \mathrm{~F}$ or each sample the shift factors, Iog aT, associated with $\mathrm{E}^{\prime}$ and $\tan \delta$ were found to be the same. In principle, a small vertical shift due to the temperature dependence of the product $\rho \mathrm{T} / \rho_{\text {ref }} \mathrm{T}_{\text {ref }}$ should also be applied, ${ }^{41}$ but in this case the correction seemed to be negligible. In the temperature interval above $T_{g}$, where the loss factor against frequency plot presents a clear maximum, its position clearly determines the shift factor $\mathrm{a}_{\mathrm{T}}$. This $\mathrm{a}_{\mathrm{T}}$ value also superposes correctly the $\mathrm{E}^{\prime}$ curve. At low temperatures, below $T_{g}$, even if there is no loss factor maximum, superposition can be performed by using the criterion of the absence of vertical shift and the equality of the $a_{\mathrm{T}}$ factor for $\tan \delta$ and $\mathrm{E}^{\prime}$. In this temperature interval scattering in the master curve appears due to the influence of the $\beta$ relaxation at high frequencies. At the same time, in this temperature interval ат becomes dependent on the thermal history due to physical aging or structural relaxation phenomenon. The temperature was decreased from the liquid state in steps of $2{ }^{\circ} \mathrm{C}$ foll owed by isothermal periods of around $30 \mathrm{~min}$ for temperature stabilization and measurements.

In the master curves it can be seen the shift of the $\alpha$-relaxation to lower frequencies (longer times) and the increase of the elastomeric plateau as the cross-linking degree increases, a behavior that is in agreement with the one detected in isochronal experiments and that could be justified with the same arguments. The similar effect of cross-linking upon the dynamic glass transition can also be detected in the dielectric relaxation spectra of polymers at fixed temperature. ${ }^{3,5}$

Also, a master curve for creep compliance was constructed for each material (Figure 3) as a function of time, taking again $\mathrm{T}_{\text {ref }}=148{ }^{\circ} \mathrm{C}$ as the reference temperature. The master curve was obtained by simple shifting of the isothermal results along the log time scale. In accordance to the DMA results, the $\alpha$-relaxation observed by creep shifts toward longer times (lower frequencies) as the cross-linking degree increases.

The shift factors above $T_{g}$, obtained during the construction of the DMA and creep master curves, were

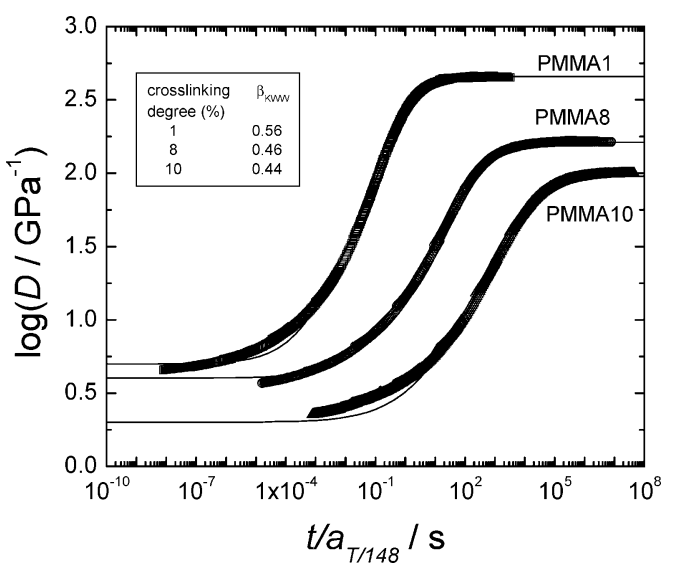

Figure 3. Master curve for creep compliance at a reference temperature of $148{ }^{\circ} \mathrm{C}$ for PMMA with different cross-linking degrees. The solid lines are the KWW fittings of the corre sponding results.
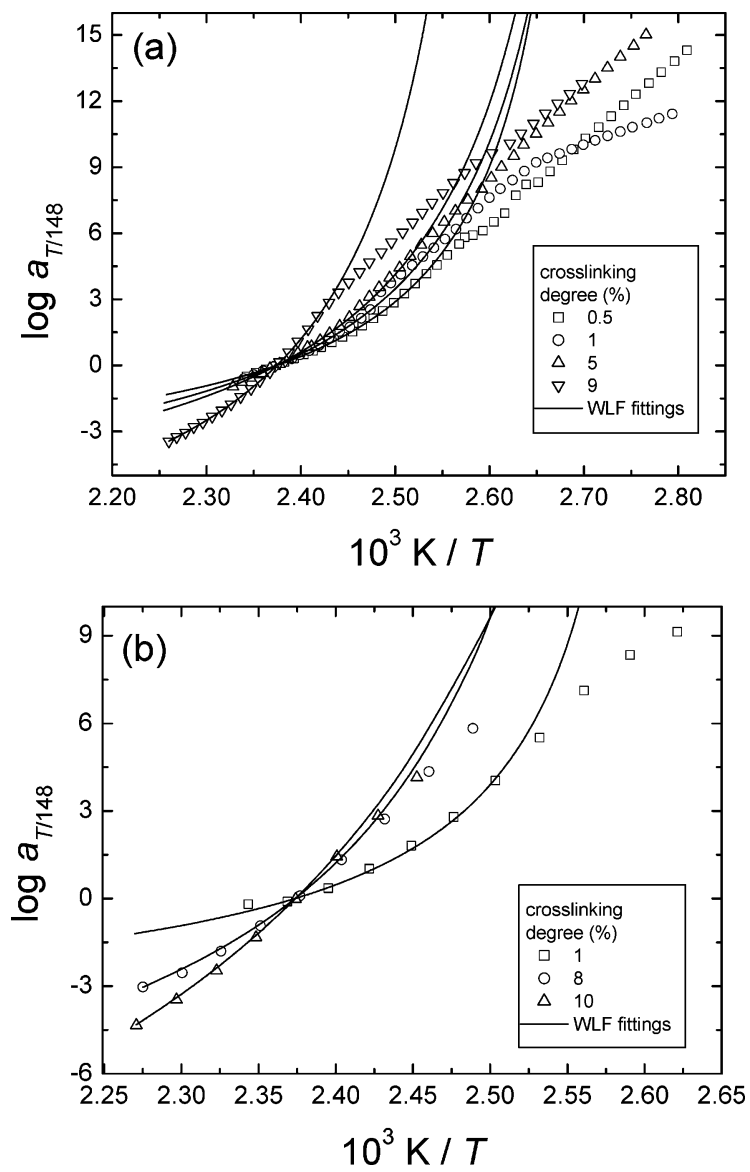

Figure 4. Temperature dependence of the shift factors for the (a) DMA and (b) creep master curves presented in Figures 2 and 3 and corresponding WLF fittings in the liquid state.

successfully described by the WLF equation ${ }^{42}$ (Figure 4)

$$
\log \mathrm{a}_{\mathrm{T}}=\log \frac{\tau(\mathrm{T})}{\tau\left(\mathrm{T}_{\mathrm{ref}}\right)}=-\frac{\mathrm{C}_{1}\left(\mathrm{~T}-\mathrm{T}_{\mathrm{ref}}\right)}{\mathrm{C}_{2}+\left(\mathrm{T}-\mathrm{T}_{\mathrm{ref}}\right)}
$$

where $C_{1}$ and $C_{2}$ depend on the material and on $T_{\text {ref. }}$. This expression usually holds for polymers over the temperature range $\mathrm{T}_{\mathrm{g}}<\mathrm{T}<\mathrm{T}_{\mathrm{g}}+100^{\circ} \mathrm{C}$ and when $\mathrm{T}_{\text {ref }}$ is identified with $\mathrm{T}_{\mathrm{g}}$ (that is not the case of this work); it was seen that $C_{1}$ and $C_{2}$ assume "universal" values close to 17.44 and $51.6^{\circ} \mathrm{C}$, respectively. ${ }^{42}$ Equation 2 is 
Table 2. WLF Parameters, VFTH Parameters, and Glass Transition Temperature $\left(T_{g}\right)$ Determined by DSC from a Heating Scan at $10^{\circ} \mathrm{C}$ min $^{-1}$ (Middle-Point Values) ${ }^{49}$ for PMMA with Different Cross-Linking Degrees

\begin{tabular}{cccrrc}
\hline $\begin{array}{c}\text { cross-linking } \\
\text { degree }(\%)\end{array}$ & \multicolumn{1}{c}{$\mathrm{C}_{1}$} & $\mathrm{C}_{2}\left({ }^{\circ} \mathrm{C}\right)$ & $\mathrm{T}_{0}\left({ }^{\circ} \mathrm{C}\right)$ & $\mathrm{B}(\mathrm{K})$ & $\mathrm{T}_{\mathrm{g}}\left({ }^{\circ} \mathrm{C}\right)$ \\
\hline 0.5 & 4.7 & 55.6 & 92.4 & 602.9 & 125 \\
1 & 6.2 & 58.9 & 89.1 & 835.9 & 127 \\
5 & 7.5 & 59.6 & 88.4 & 1034.4 & 133 \\
9 & 10.5 & 43.8 & 104.2 & 1059.4 & 144
\end{tabular}

equivalent to the Vogel-Fulcher-Tamman-Hesse equation (VFTH): $31-33$

$$
\tau(\mathrm{T})=\tau_{0} \exp \left(\frac{\mathrm{B}}{\mathrm{T}-\mathrm{T}_{0}}\right), \quad \mathrm{T}_{0}<\mathrm{T}_{\mathrm{g}}
$$

where $\tau_{0}$ is a preexponential factor and $\mathrm{B}$ and $\mathrm{T}_{0}$ are specific adjustable parameters. The WLF and VFTH equations are the most frequently models applied for describing the non-Arrhenius behavior. The WLF and VFTH parameters are related by $\mathrm{C}_{1}=\mathrm{B} / 2.303\left(\mathrm{~T}_{\text {ref }}-\right.$ $\mathrm{T}_{0}$ ) and $\mathrm{C}_{2}=\mathrm{T}_{\text {ref }}-\mathrm{T}_{0 .}{ }^{41}$ The WLF and VFTH parameters for the studied PMMAs are presented in Table 2 as a function of the cross-linking degree, calculated from DMA results.

In this work the isothermal results obtained by both DMA and creep were analyzed according to the KWW model. 29,30 The time-distribution function defined by this model is

$$
\phi(\mathrm{t})=\exp \left[-\left(\frac{\mathrm{t}}{\tau}\right)^{\beta_{\mathrm{kww}}}\right]
$$

where $0<\beta_{\mathrm{KWW}} \leq 1$ and $\tau$ is the KWW relaxation time. The $\beta_{\mathrm{k} w w}$ parameter is associated with the distribution of characteristic times, a small value of $\beta_{\mathrm{Kww}}$ implies a broad distribution, and $\beta_{k w w}$ close to unity implies a narrow distribution (an exponential response). This model was proposed as an empirical model although providing an excellent fit to the shape of the relaxation curves obtained from different techniques and different materials.

Some years ago $\mathrm{Ngai}$ and co-workers have proposed a model, known as the coupling model, ${ }^{43-45}$ that results in an expression for $\phi(t)$ that is similar to the KWW model:

$$
\phi(\mathrm{t})=\exp \left[-\left(\frac{\mathrm{t}}{\tau^{*}}\right)^{1-\mathrm{n}}\right]
$$

where $\tau^{*}$ is the effective (observed) relaxation time and $\mathrm{n}$ is related to the intermolecular coupling between the relaxing species. The coupling model also predicts a relation between $\tau^{*}$ and the intermolecularly uncorrelated relaxation time $\tau_{0}: 43$

$$
\tau^{*}=\left([1-\mathrm{n}] \mathrm{t}_{\mathrm{c}}^{-\mathrm{n}} \tau_{0}\right)^{1 /(1-\mathrm{n})}
$$

where $t_{c}$ is the time after which the intermolecular constraints become manifest. $\tau_{0}$ has a magnitude that depends on the local friction.

For DMA or dielecric spectroscopy results it is not easy to obtain an accurate value of $\beta_{\mathrm{KWW}}$ for PMMA because there is a large degree of overlap of the $\alpha$ and $\beta$-processes, ${ }^{46}$ the $\beta$-process being especially broad. This $\beta$-relaxation has been assigned to the hindered rotation of the $-\mathrm{COOCH}_{3}$ group about the $\mathrm{C}-\mathrm{C}$ bond linking it to the main chain. ${ }^{37,47}$ It has been found that the

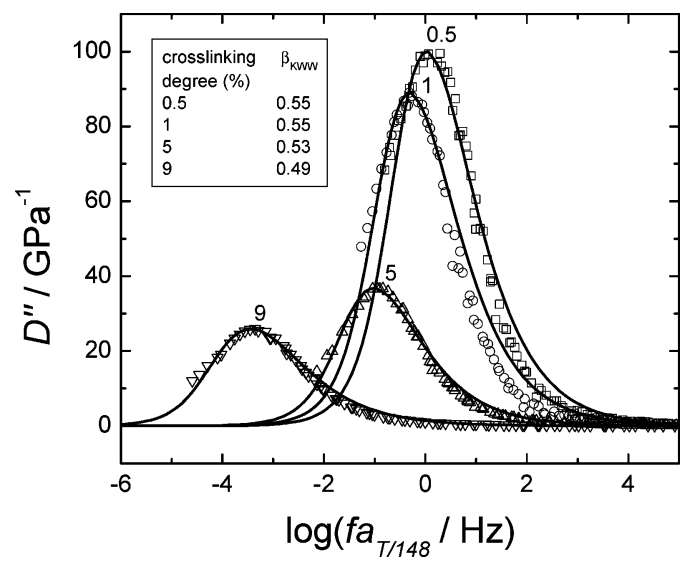

Figure 5. Loss compliance ( $\left.D^{\prime \prime}\right)$ master curves at a reference temperature of $148{ }^{\circ} \mathrm{C}$ for PMMA with different cross-linking degrees. For clarity, not all experimental points are repre sented. The solid lines are the fittings with the KWW function. The values of the $\beta_{\mathrm{K} w \mathrm{w}}$ parameter obtained for the distinct samples are also shown in this figure.

position of this relaxation is independent of the crosslinking degree 20 due to the nature of the motions involved (localized motions) and depends only on the size of the lateral group. These findings may be generalized, and other authors have also noticed that the local motions of polymer chains are not influenced by the cross-linking degree. ${ }^{5}$

For the DMA data, we selected to fit the loss compliance $\left(D^{\prime \prime}\right)$ to the KWW function because in the curves of $E^{\prime}$ (Figure 2 ) the glassy plateau is not well-defined and in the $E^{\prime \prime}$ curves (not shown) the maximum of the peak is also not very clear. The shift factors for $E^{\prime}, E^{\prime \prime}$, $\tan \delta$, and D" were found to be approximately the same for each sample, especially for the samples with higher cross-linking degree. Therefore, D" master curves were obtained by simple shifting of the isothermal experimental results, and by this way curves with well-defined peaks were obtained (Figure 5).

The mechanical compliance $\mathrm{D}^{*}(\omega)=\mathrm{D}^{\prime}(\omega)-\mathrm{iD}^{\prime \prime}(\omega)$ is given by the one-sided Fourier or pure imaginary Laplace transform of the time derivative of the decay function $\phi(\mathrm{t}) \mathbf{4}^{48}$

$$
\frac{D^{*}(\omega)-D_{u}}{D_{r}-D_{u}}=\int_{0}^{\infty}\left[-\frac{d \phi(t)}{d t}\right] \exp (-i \omega t) d t
$$

where $\mathrm{u}$ and $\mathrm{r}$ designate unrelaxed (for $\omega \rightarrow \infty$ or $\mathrm{t} \rightarrow 0$ ) and relaxed compliance (for $\omega \rightarrow 0$ or $\mathrm{t} \rightarrow \infty$ ) values, respectively, and $\mathrm{i}^{2}=-1$.

In Figure 5 are also shown the fittings according to the KWW model, using eqs 4 and 7 .

For low values of $\beta_{\mathrm{KWw}}$ the asymmetry of the $\mathrm{E}^{\prime \prime}$ and $D^{\prime \prime}$ peaks is very noticeable. However, these results show that the experimental master curves are more symmetric about the peak than the theoretical KWW curves. This observation is in agreement with the al ready mentioned results found by Glatz-Reichenbach and co-workers, ${ }^{5}$ where the KWW asymmetry was also depressed for cross-linked poly(vinylethylene) samples. This could suggest that, while the majority of the processes contributing to the $\alpha$-relaxation behave according to the KWW model, there are also slower processes overlapping the main component of the $\alpha$ process; the source of these additional contributions is the segmental processes of groups close to or strongly influenced by the cross-links. In addition, it should exist 


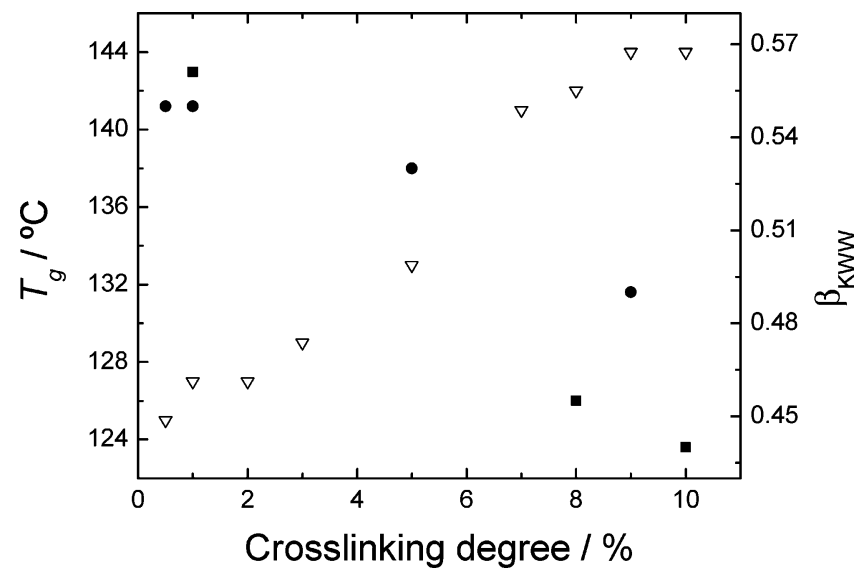

Figure 6. Dependence of the glass transition temperature and $\beta_{\mathrm{K} w \mathrm{w}}$ parameter on the concentration of EGDMA in the network: $(\nabla) \mathrm{T}_{\mathrm{g}}$ determined by DSC from a heating scan at $10^{\circ} \mathrm{C} \mathrm{min}^{-1}$ (middle-point values); ${ }^{49}(\bullet) \beta_{\mathrm{Kww}}$ determined from DMA experiments; (ם) $\beta_{\mathrm{kww}}$ from creep experiments.

different degrees of superposition with the $\beta$-relaxation at higher frequencies, and hence, the high-frequency side of the $\alpha$-peak also deviates from the predictions.

The creep data were al so fitted to the KWW function. The relation between the creep compliance $\mathrm{D}(\mathrm{t})$ and the time distribution function $\phi(\mathrm{t})$ is

$$
\frac{D(t)-D_{u}}{D_{r}-D_{u}}=1-\phi(t)
$$

The fitted curves are shown in Figure 3. In this case, because of the superposition with the $\beta$-relaxation, the low time side of the data is not well fitted.

It should be pointed out that, as it was observed in this work, the $\alpha$-relaxation of cross-linked PMMA shifts to lower frequencies (higher temperatures) as the crosslinking degree increases, and supposing that the $\beta$-relaxation is not affected by cross-linking, one should expect that the samples with higher cross-linking degree would present the lowest overlap between the $\beta$ and $\alpha$ processes.

The $\beta_{\mathrm{K} w w}$ values as a function of the cross-linking degree are presented in Figure 6 for both creep and DMA data. It is observed a decrease of $\beta_{\mathrm{Kww}}$ as the cross-linker content increases, from 0.55 to 0.44 when the cross-linker concentration varies from 0.5 to $10 \%$. This provides an indication that the characteristic times distribution tends to broaden as the cross-linking degree increases. The cal orimetric glass transition temperature $T_{g}$ vs cross-linking degree, determined from previous DSC measurements 49 at $10^{\circ} \mathrm{C} \mathrm{min}-1$, is also presented in Figure 6. It is evident the pronounced variation of $\mathrm{T}_{\mathrm{g}}$ with cross-linking density $\left(\approx 20^{\circ} \mathrm{C}\right)$ from the slightly cross-linked PMMA (PMMA0.5) to the more cross-linked sample (PMMA10).

The same variation of the $\beta_{\mathrm{Kww}}$ parameter with crosslinking degree has been detected for other cross-linked polymers. ${ }^{3,5,7,8}$ As has been pointed out by Kramarenko et al., ${ }^{3}$ the decrease of $\beta_{\mathrm{KWw}}$ as the cross-linking degree increases could be explained in terms of the coupling model mentioned before, being a consequence of the increase of the intermolecular coupling between the rel axing species originated by the additional constraints introduced by cross-linking and leading to a more pronounced non-Debye behavior.
The $\beta_{\mathrm{K} w W}$ values obtained are higher than the ones found in the literature for un-cross-linked PMMA, $\beta_{\mathrm{Kww}}$ $\approx 0.33,50$ but normally these are values obtained from fittings of $E^{\prime}$ or $E^{\prime \prime}$, and also, because of cross-linking, the response is more symmetric than would be for an un-cross-linked material, as discussed before. It should be pointed in this context that $E^{\prime}$ (or $E^{\prime \prime}$ ) and $D^{\prime}$ (or $D^{\prime \prime}$ ) reflect two different times scales, the former being linked to the relaxation times and the latter is associated with retardation times. In a previous work ${ }^{51}$ it was shown for PM MA5 a different temperature dependence of retardation and relaxation times, and this peculiar behavior was attributed to the overlap of the $\alpha$ and $\beta$ relaxations. The superposition of these two transitions is a complex process as has been noticed elsewhere $\mathrm{e}^{52}$ and for higher temperatures or frequencies originates just one relaxation designated as $\alpha \beta$ process, ${ }^{53}$ which is not just a mere superposition of the two individual relaxations. Nevertheless, for the low frequencies used in this work, the $\beta$ and $\alpha$ relaxation are two independent relaxation processes that partially overlap each other. We assume here that the influence of the $\beta$ relaxation would be distinct for the compliance and elastic modulus curves, being smaller in the case of the creep compliance curve which would conduct to a distinct temperature dependence of the retardation and the relaxation times and would also lead to different $\beta_{\mathrm{K} w \mathrm{w}}$ values if compliance is fitted instead of modulus.

3.2. TSR Results. PMMA0.5, PMMA5, and PMMA9 were studied by TSR in the glass transition region using the thermal sampling (TS) procedure. The creep temperatures range were 51.3-156.8, 71.4-151.4, and 69.4-164.8 ${ }^{\circ} \mathrm{C}$ for PMMA0.5, PMMA5, and PMMA9, respectively. Some of the $\epsilon$ vS T curves obtained are shown in Figure 7. For all the samples the curves are shifted to higher temperatures, and the low-temperature plateau of the TS curves increases as $\mathrm{T}_{\sigma}$ increases. This behavior was al ready explained el sewhere: ${ }^{36}$ during the increase of $\mathrm{T}_{\sigma}$ in the glassy state the number of the activated modes in a TS experiment increases because one approaches the maximum of the distribution of retardation times, which occurs near $\mathrm{T}_{\mathrm{g}}$. Therefore, it is expected an increase of $\epsilon_{0}$ when $\mathbf{T}_{\sigma}$ increases in the glassy state.

The anelastic response associated with each TS curve is usually analyzed as a quasi-elementary process, often modeled by a Voigt-Kelvin model. The temperature dependence of the retardation times of such curves are obtained up to near the temperature of maximum strain rate using the so-called Bucci method:

$$
\tau(\mathrm{T})=\frac{\epsilon(\mathrm{T})}{\beta|\mathrm{d} \epsilon(\mathrm{T}) / \mathrm{dT}|}
$$

where $\beta$ is the heating rate. Usually it is assumed an Arrhenius dependence of $\tau(\mathrm{T})$ which permits to obtain both the activation energy, $E_{a}$, and the preexponential factor, $\tau_{0}$, of each TS curve. More details about the calculation of the thermokinetic parameters may be found in a previous work. ${ }^{35}$

The variation of $\mathrm{E}_{\mathrm{a}}$ with $\mathrm{T}_{\sigma}$ for the three samples is presented in Figure 8. For all the samples it was possible to obtain $E_{a}$ at quite low temperatures, far bel ow $T_{g}$. In this temperature range $E_{a}$ depends only slightly on temperature, adopting an Ahrrenius behavior characteristic of the glassy state. However, it was demonstrated, using the activation entropy concept, that 

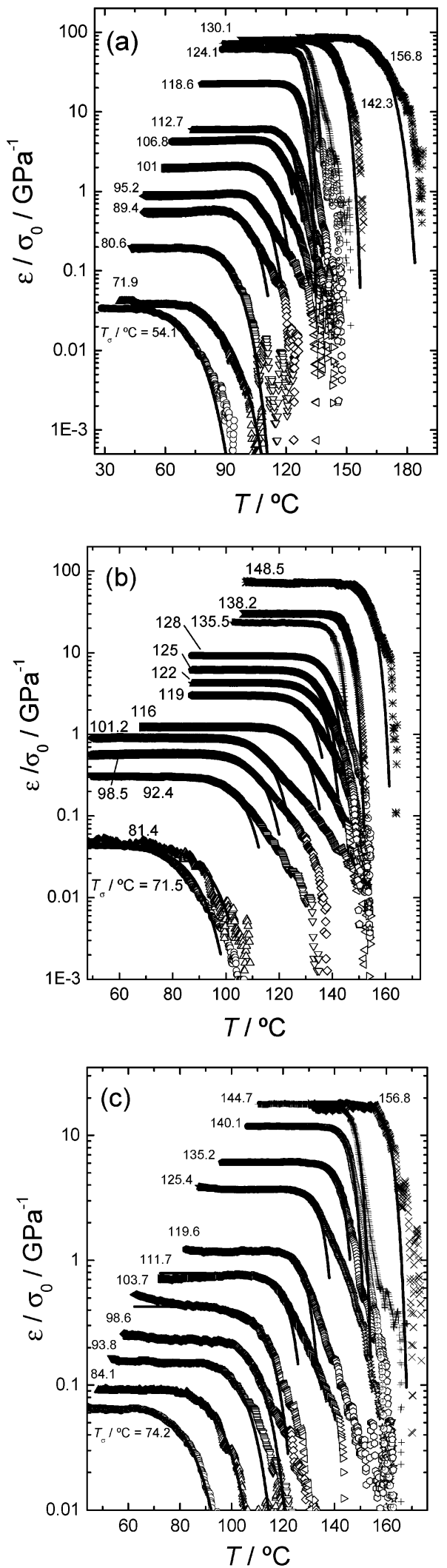

Figure 7. TS results in the glass transition region for (a) PMMA with $0.5 \%$ of EGDMA, (b) PMMA with $5 \%$ of EGDMA, and (c) PMMA with 9\% of EGDMA, obtained at different creep temperatures $\mathrm{T}_{\sigma}$ (in the graphics). The solid lines are the simulated curves with the thermokinetic parameters obtained from the Arrhenius fitting of the $\tau(\mathrm{T})$ results.

even at these lower temperatures the involved motions in PMMA are still cooperative. ${ }^{28}$ Such behavior seems to be quite general, as in a previous study in polycarbonate was also found at low temperatures. ${ }^{54}$ For higher
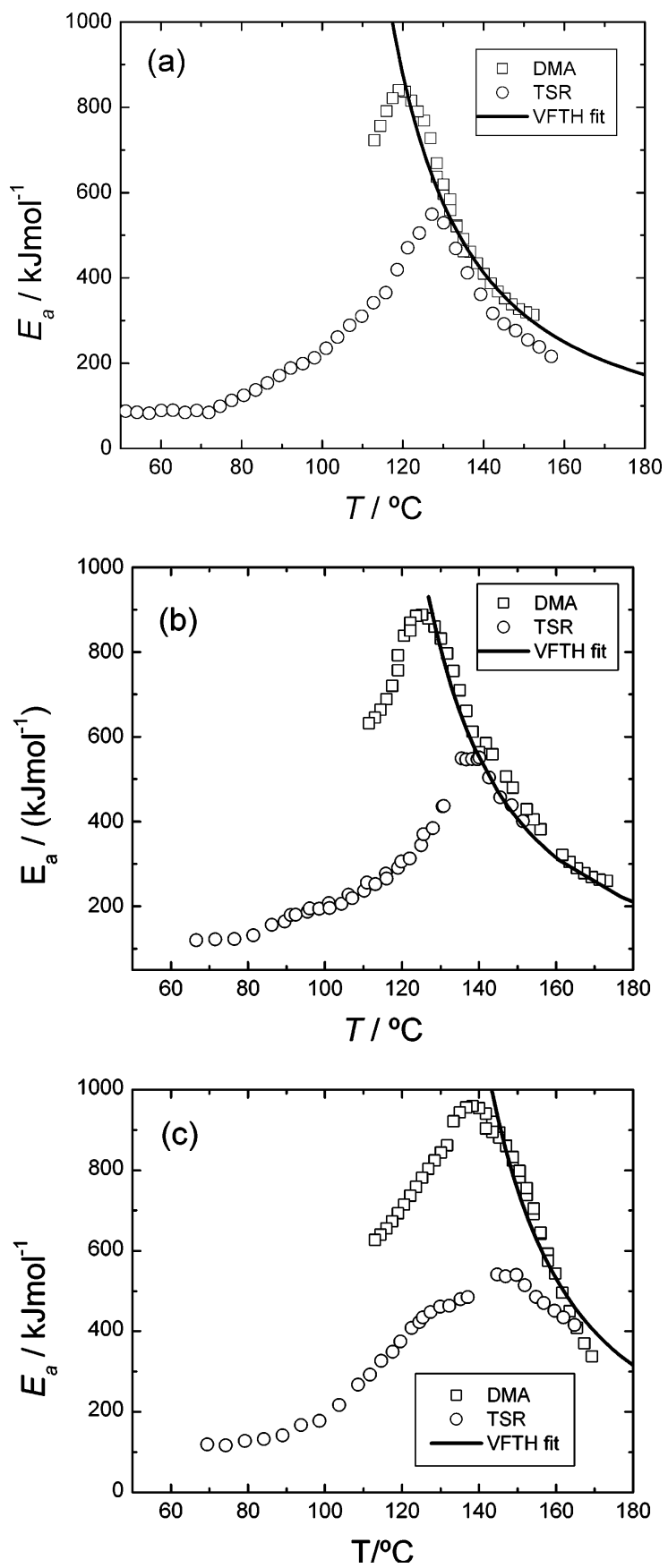

Figure 8. Temperature dependence of the apparent activation energy across the glass transition for (a) PMMA with $0.5 \%$ of EGDMA, (b) PMMA with $5 \%$ of EGDMA, and (c) PMMA with $9 \%$ of EGDMA. The squares are for the DMA results, calculated from the temperature shift factors (numerical differentiation). By fitting the shift factors in the liquid state with the WLF equation and using the relations between the WLF and VFTH parameters $E_{a}(T)$ curves were computed with the corresponding $B$ and $T_{0}$ parameters (solid lines). The circles are for the TSR results, where $E_{a}$ was obtained from the TS curves using eq 9 and assuming an Arrhenius behavior.

temperatures, $E_{a}$ increases with the increase of temperature, reflecting the approach to the VFTH regime and the cooperative motions of the $\alpha$-relaxation. Above $\mathrm{T}_{\mathrm{g}}$ it is observed a decrease of $\mathrm{E}_{\mathrm{a}}$ as $\mathrm{T}_{\sigma}$ increases, a behavior typical of the VFTH regime. Therefore, a maximum in $E_{a}(T)$ occurs naturally at temperatures around $T_{g}$, due to the transition between the Arrhenius and the VFTH regimes. The same behavior was detected previously by TSR on a semicrystalline poly(ethylene terephthalate). ${ }^{36}$ Note that the temperatures of the 


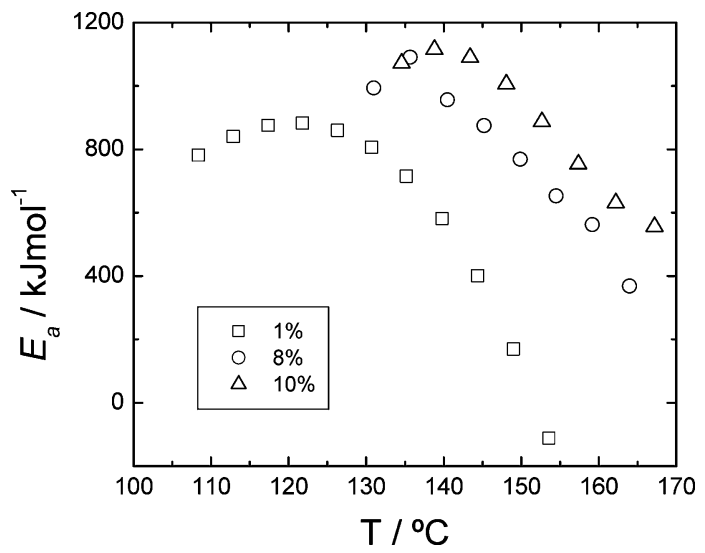

Figure 9. Temperature dependence of the apparent activation energy across the glass transition for PMMA with $1 \%$ of EGDMA $(\triangle)$, PMMA with $8 \%$ of EGDMA $(\bigcirc)$, and PMMA with $10 \%$ of EGDMA $(\Delta)$ obtained from creep data (Figure 3 ).

maximum $E_{a}$ for the distinct networks (Figure 8) are close to the calorimetric $T_{g}$ values (Figure 6). This can be explained by the similar equivalent frequencies associated with the TSR and DSC techniques $\left(\sim 10^{-2}\right.$ $\mathrm{Hz}$ ).

The same features in $E_{a}(T)$ are observed by thermally stimulated depolarization currents (TSDC) (e.g., refs 28, 55 , and 56). It is interesting to note that the values of maximum $\mathrm{E}_{\mathrm{a}}$ obtained by TSR in this work, $\mathrm{E}_{\mathrm{a}} \approx 549$ $\mathrm{kJ} / \mathrm{mol}$ for all samples, are similar to the ones found in the literature for PMMA by thermally stimulated creep $(\approx 590 \mathrm{~kJ} / \mathrm{mol})^{28}$ and TSDC $(\approx 350 \mathrm{~kJ} / \mathrm{mol}) .{ }^{57}$ Also, the temperatures of maximum $\mathrm{E}_{\mathrm{a}}$ obtained by TSDC $(\approx 120$ $\left.{ }^{\circ} \mathrm{C}\right)^{58}$ for un-cross-linked conventional PMMA, using the TS procedure, is similar to the one found by us for the slightly cross-linked sample $\left(\approx 127.2^{\circ} \mathrm{C}\right)$, although our value is somewhat higher due to the reaction with EGDMA.

The temperature dependence of $E_{a}$, calculated from the $a_{T}$ shift factors obtained from DMA and creep experiments, is also represented in Figures 8 and 9, respectively. As can be observed in Figure 4, the Arrhenius plot (logarithm of the relaxation times against the reciprocal of the temperature) around the $\alpha$-relaxation exhibits a change upon the occurrence of the glass transition. In the equilibrium liquid state, the trends follow the VFTH or WLF regime, where the slope of the $\log \tau$ vs $1 / T$ diagram continuously increases with decreasing temperature. As a consequence, the apparent activation energy $E_{a}(T)$

$$
E_{a}(T)=R \ln (10) \frac{d \log a_{T}}{d(1 / T)}=R \frac{d \ln \langle\tau\rangle}{d(1 / T)}
$$

increases with decreasing temperature in the liquid state. When the glass transition takes place, the relaxation times start deviating from the VFTH or WLF curve, and in the glassy state they adopt, at any temperature, values higher than those corresponding to the equilibrium liquid at the same temperature. As the deviation of the position of the relaxation curve with respect to the VFTH behavior is much greater than the uncertainty in the determination on $\mathrm{a}_{\mathrm{T}}$ (see Figure 4), it is believed that the peak in the $\mathrm{E}_{\mathrm{a}}$ curve and the marked decrease in $E_{a}$ below $T_{g}$ cannot be attributed to a failure of the frequency-temperature superposition or to volume relaxation effects.
Thus, the transition of the temperature dependence of the characteristic times between the VFTH and the Arrhenius behavior when the glass transition takes place can be observed by TSR as well as by DMA or creep experiments; the main difference is that TSR allows to characterize more accurately the characteristic times in the glassy state at temperatures up to $60 \mathrm{deg}$ bel ow $T_{g}$, but the experiments can be extended only to 20 deg above $\mathrm{T}_{\mathrm{g}}$. On the contrary, DMA or creep experiments allow to characterize the main relaxation only a few degrees below $T_{g}$, but experiments can be performed up to 50 deg above $T_{g}$, thus covering mostly the VFTH regime. In the TSR experiments the transition from the Arrhenius behavior to the VFTH behavior is shifted toward higher temperatures with respect to the data obtained in DMA and creep experiments, and consequently the maximum value of $E_{a}$ is lower in TSR than in DMA. This may be a consequence of the isothermal character of the DMA experiments that allow keeping the materials in thermodynamic equilibrium down to lower temperatures.

Above $T_{g}$ the $E_{a}$ values obtained from DMA, creep, and TS experiments agree very well. This is due to the fact that assuming a thermorheol ogically simple system the slope of $\log \langle\tau\rangle$ vs $1 / T$ (from the DMA data) is similar to the slope of the TS curves in an Arrhenius diagram obtained at the same temperature, although shifted through higher times. ${ }^{51}$

The effect of the cross-linking degree in the TS results is also evident in Figure 8: an increase of the crosslinking degree leads to a shift of the maximum $E_{a}$ to higher temperatures, which means that the $\alpha$-relaxation of PMMA is also shifted to higher temperatures as the content of EGDMA increases. The temperature of maximum $E_{a}$ changes $\approx 19{ }^{\circ} \mathrm{C}$ from PMMA0.5 to PMMA9, so a significant displacement of the $\alpha$-relaxation occurs, this displacement being analogous to the ones detected by DMA and creep. Moreover, a broader transition from the Arrhenius behavior to the Vogel behavior is observed as the cross-linking density is getting higher, which means that the $\alpha$-relaxation broadens as the EGDMA content increases due to the increase of molecular environments felt by the segmental motions, imposed by the cross-links. This is particularly evident when the results of PMMA0.5 (F igure 8a) and PMMA9 (Figure $8 \mathrm{c}$ ) are compared. The $\mathrm{E}_{\mathrm{a}}(\mathrm{T})$ curve obtained by TSR is broader for PMMA9 than for PMMA05. This behavior is in agreement with the variation of $\beta_{\mathrm{K} w w}$ with cross-linking degree obtained from the fittings of DMA and creep results and has already been explained on the basis of the coupling model.

3.3. F ragility. Fragility is related to the magnitude of the decrease of $\log \tau$ (or $\log \mathrm{a}_{\mathrm{T}}$ ) with decreasing $\mathrm{T}_{\mathrm{g}} /$ $\mathrm{T}^{59-61}$ and thus may be parametrized by the steepness "index" m:

$$
\mathrm{m}=\left.\frac{\mathrm{d} \log \tau}{\mathrm{d}\left(\mathrm{T}_{\mathrm{g}} / \mathrm{T}\right)}\right|_{\mathrm{T}=\mathrm{T}_{\mathrm{g}}}=\left.\frac{\mathrm{d} \log \mathrm{a}_{\mathrm{T}}}{\mathrm{d}\left(\mathrm{T}_{\mathrm{g}} / \mathrm{T}\right)}\right|_{\mathrm{T}=\mathrm{T}_{\mathrm{g}}}
$$

The value $m=16$ corresponds to Arrhenius behavior ("strong" limit), and for $m>200$ the systems reach the "fragile" limit. ${ }^{62}$ Using eqs 2 and 3, m may be directly obtained from the VFTH or WLF parameters (when $T_{\text {ref }}$ $\left.\neq \mathrm{T}_{\mathrm{g}}\right)$ : 


$$
\mathrm{m}=\frac{\mathrm{BT} g / 2.303}{\left(\mathrm{~T}_{\mathrm{g}}-\mathrm{T}_{0}\right)^{2}}=\frac{\mathrm{T}_{\mathrm{g}} \mathrm{C}_{1} \mathrm{C}_{2}}{\left(\mathrm{C}_{2}+\mathrm{T}_{\mathrm{g}}-\mathrm{T}_{\text {ref }}\right)^{2}}
$$

where $C_{1}$ and $C_{2}$ are the coefficients of the WLF equation referred to $T_{\text {ref. }} \mathrm{m}$ may be also obtained directly from the $E_{a}(T)$ plot, such as the one shown in Figure 8, from

$$
m=E_{a}\left(T_{g}\right) /\left[\ln (10) R T_{g}\right]
$$

In this work the fragility index of the PMMA networks was calculated both from isothermal DMA and creep experiments by applying eq 13. $\mathrm{T}_{\mathrm{g}}$ is the calorimetric glass transition temperature, a commonly used definition in the literature. As the DSC technique has a low equivalent frequency, these values should be similar to the ones corresponding to the also common definition of $\mathrm{T}_{\mathrm{g}}$ : the temperature at which the relaxation time is $100 \mathrm{~s}$. For PMMA0.5, PMMA5, and PMMA9 and from isothermal DMA results the values of $E_{a}$ at $T_{g}$ are 760,780 , and $925 \mathrm{~kJ} \mathrm{~mol}^{-1}$, respectively, and the obtained $m$ values are МРMмA0.5 $=99$, MPMмA5 $=100$, and mPMMA9 $_{\text {P }}=116$. For PMMA1, PMMA8, and PMMA 10 and from creep results the values of $E_{a}$ at $T_{g}$ are 850, 1000, and $1100 \mathrm{~kJ} \mathrm{~mol}^{-1}$, respectively, and $m_{\text {PMMA1 }}=111, m_{\text {PMMA }}=126$, and $m_{\text {PMMA } 10}=138$.

So, an increase of $\mathrm{m}$ with increasing cross-linking degree was obtained by either DMA and creep. Our values are in agreement with the ones found in the literature for un-cross-linked PMMA by DSC $(\mathrm{m} \approx$ $103)^{63}$ and by mechanical spectroscopy $(\mathrm{m} \approx 145) .{ }^{64}$ Of course that, as discussed in a previous work, ${ }^{51}$ the $\mathrm{m}$ values are dependent on the technique used to evaluate fragility. In this work ${ }^{51}$ it was shown that, due to the particular thermal profile of TSR, lower values of $m$ would be obtained by this technique, relative to the ones obtained by isothermal spectroscopies.

The detected variation of $m$ reveals that fragility increases with increasing cross-linking degree. Therefore, the non-Arrhenius character of the temperaturedependent behavior is more pronounced for the samples with higher content of EGDMA. This could be attributed to the constraints in the segmental mobility imposed by the cross-links. The increase of fragility with increasing cross-linking degree has been also detected in other materials. ${ }^{3,8}$ In ref 8 dielectric spectroscopy was used to study the segmental relaxation in poly(vinylethylene) networks. The observed changes in the Angell plot (log $\tau$ or a related property such as the shear viscosity $\eta$ vs $\mathrm{T}_{\mathrm{g}} / \mathrm{T}$ ) and in fragility were primarily attributed to a stronger intermolecular coupling. On the contrary, the Angell plot for samples with different degree of crystallinity showed that the temperature dependence of segmental relaxation times is independent of the degree of crystallinity. ${ }^{65}$ This is an interesting feature because crystallization originates similar changes on segmental relaxation as cross-linking: increase of $\mathrm{T}_{\mathrm{g}}$ and broader relaxation, ${ }^{66}$ but the origin of these changes should be different for these two factors as implied by the previous considerations. In fact, by application of the coupling model it was shown ${ }^{8}$ that the constraints on segments in proximity to crystallites increase their relaxation time; i.e., crystallization primarily affects $\tau_{0}$ (see eq 6), but the degree of intermolecular coupling (n) for most chains is unaffected, so the change of the peak frequency is altered mainly as a consequence of increased local friction. This is precisely the opposite that occurs in cross-linked materials where an increase of cross-linking density is accompanied by a slight increase of $\tau_{0}$ and a stronger intermolecular coupling (higher $\mathrm{n}$ ). ${ }^{8}$

The fragility has been related with the non-Debye behavior. ${ }^{62}$ It has been found for a large number of polymers, ionic melts, supercooled molecular liquids, and covalent glass formers that as $m$ increases, the exponent $\beta_{\mathrm{K} w \mathrm{w}}$ decreases, which means that fragile systems usually are described by more nonexponential relaxation functions. These results are in agreement with the coupling model that predicts a more exponential relaxation in glass formers in which due to a relatively simple molecular structure the coupling is reduced. In our case we al so found higher values of $\beta_{\mathrm{K} w \mathrm{w}}$ as $m$ decreases.

It should be pointed out that the fragility discussed in this work is the so-called kinetic or dynamic fragility, related to the relaxational manifestations and not the thermodynamic fragility quantified in several ways. Usually it is obtained by the step change on the heat capacity of the glass transition $\Delta \mathrm{C}_{\mathrm{p}}\left(\mathrm{T}_{\mathrm{g}}\right)^{67}$ or by the ratio between the absolute heat capacities in the liquid and glassy states $\left(C_{p}^{1} / C_{p}{ }^{9}\right),{ }^{64}$ but also by changes on the coefficient of thermal expansion ${ }^{68}$ or on the excess entropy. ${ }^{69}$ In this context all the PMMA samples analyzed can be classified, according to the fragility scale, as kinetically fragile systems with a non-Arrhenius transport behavior as usual in polymeric systems. ${ }^{62}$

\section{Conclusions}

The study of PMMA networks cross-linked with different amounts of EGDMA by dynamic mechanical analysis (DMA), creep, and thermally stimulated recovery (TSR) reveal distinct but complementary aspects of the $\alpha$-relaxation of these systems. The main aspects are now summarized:

1. DMA ( $\mathrm{E}^{\prime}, \tan \delta$, and $\left.\mathrm{D}^{\prime \prime}\right)$ and creep compliance $(\mathrm{D}(\mathrm{t}))$ master curves for PMMA networks cross-linked with different amounts of EGDMA were successfully obtained by horizontal shifting of the isothermal experiments. It was detected a shift of the $\alpha$-relaxation to lower frequencies, the increase of the elastomeric plateau, and the broadening of the relaxation as the cross-linking degree increases.

2. The loss compliance and the creep compliance were fitted to the KWW equation. The fittings revealed a more symmetric response of these cross-linked samples and the influence of the $\beta$-relaxation at higher frequencies. It was observed a decrease of $\beta_{\mathrm{K} w w}$ as the crosslinker content increases, from 0.55 to 0.44 when the cross-linker concentration varies from 0.5 to $10 \%$, reflecting a broadening of the relaxation with increasing cross-linking degree.

3. The $\beta_{\mathrm{K} w \mathrm{w}}$ values obtained in this work were higher than the ones found in the literature from fittings of un-cross-linked PMMA, where E' or E" data are usually used. This was mainly attributed to the distinct influence of the $\beta$ relaxation in the compliance and elastic modulus curves, being smaller in the case of the compliance curve.

4. TSR results also clearly revealed a significant shift of the $\alpha$-relaxation to longer times as well as a broadening of the process as the cross-linking degree increases. A variation of $\mathrm{T}_{\mathrm{g}}$ with cross-linking degree of about 20 ${ }^{\circ} \mathrm{C}$ from the slightly cross-linked PMMA to the more cross-linked sample was observed for the studied networks by all techniques. 
5. A transition from a VFTH to an Arrhenius behavior was clearly observed by the three mechanical spectroscopy experiments. The main difference is that TSR allows to characterize more effectively the relaxation features in the glassy state.

6. The effect of cross-linking density on the fragility of these PMMA networks was studied. The higher values of the fragility index $\mathrm{m}$ obtained for these systems (between 99 and 138) indicated that they are kinetically fragile systems. Moreover, it was found that $m$ increases with increasing cross-linking degree and that $\beta_{\mathrm{K} w w}$ also increases as $\mathrm{m}$ decreases. This behavior was attributed to a stronger intermolecular coupling.

Acknowledgment. Financial support for this work was provided by FCT, through the POCTI and FEDER programs. N.M.A. acknowledges FCT for the financial support through Grant PRAXIS XXI/BD/20327/99. J .A.G.T. and J .L.G.R. acknowledge the support of the Spanish Science and Technology Ministry through the MAT2001-2678-C02-01 project.

\section{References and Notes}

(1) Matsuoka, S. Relaxation Phenomena in Polymers; Hanser: Munich, 1992

(2) Ngai, K. L.; Rendell, R. W. J . Non-Cryst. Solids 1991, 131$133,942$.

(3) Kramarenko, V. Y.; Ezquerra, T. A.; Sics, I.; Baltá-Calleja, F. J .; Privalko, V. P. J . Chem. Phys. 2000, 113, 447.

(4) Casalini, R.; Fioretto, D.; Livi, A.; Lucchesi, M.; Rolla, P. A. Phys. Rev. B 1997, 56, 3016.

(5) Glatz-Reichenbach, J. K. W.; Sorriero, L.J .; Fitzgerald, J . J . Macromolecules 1994, 27, 1338.

(6) Ilavský, M.; Hasa, J .; J anácek, J . Int. J . Polym. Mater. 1972 $1,187$.

(7) Prochazka, F.; Durand, D.; Nicolai, T. J . Rheol. 1999, 43, 1511.

(8) Roland, C. M. Macromolecules 1994, 27, 4242.

(9) Gsell, C.; Souahi, A. J . Eng. Mater. Technol. 1997, 119, 223.

(10) Rubinstein, M.; Panyukov, S. Macromol ecules 2002, 35, 6670.

(11) Kuhn, W. J . Polym. Sci. 1946, 1, 380.

(12) Wall, F. T.; Flory, P. J . J . Chem. Phys. 1951, 19, 1435.

(13) J ames, H. M. J . Chem. Phys. 1947, 15, 651.

(14) Guth, E. J . Chem. Phys. 1948, 11, 455.

(15) de Gennes, P.-G-. J . Chem. Phys. 1971, 55, 572.

(16) Doï, M.; Edwards, S. F. J . Chem. Soc., Faraday Trans. 2 1978, 74, 1789.

(17) Doï, M.; Edwards, S. F. J . Chem. Soc., Faraday Trans. 21979 $75,38$.

(18) Perez, J .; Muzeau, E.; Cavaillé, J . Y. Plast. Rubb. Compos. Applic. 1992, 18, 139.

(19) Hempel, E.; Beiner, M.; Huth, H.; Donth, E. Thermochim. Acta 2002, 391, 219.

(20) Salmerón Sánchez, M.; Gallego Ferrer, G.; Torregrosa Cabanilles, C.; Meseguer Dueñas, J. M.; Monleón Pradas, M.; Gómez Ribelles, J. L. Polymer 2001, 42, 10071.

(21) Hou, S. S.; Kuo, P. L. Polymer 2001, 42, 9505.

(22) Mano, J . F. Thermochim. Acta 1999, 332, 161

(23) Mudarra, M.; J oumha, A.; Belana, J .; Toureille, A. Polymer 1999, 40, 6977

(24) Gourari, A.; Bendaoud, M.; Lacabanne, C.; Boyer, R. F. J . Polym. Sci., Polym. Phys. Ed. 1985, 23, 889.

(25) Lacabanne, C.; Chatain, D. J . Polym. Sci., Polym. Phys. Ed. 1973, 11, 2315.

(26) Dufresne, A.; Etienne, S.; Perez, J .; Demont, P.; Diffalah, M.; Lacabanne, C.; Martinez, J. J . Polymer 1996, 37, 2359.

(27) Doulut, S.; Bacharan, C.; Demont, P.; Bernès, A.; Lacabanne, C. J . Non-Cryst. Solids 1998, 235-237, 645.
(28) Doulut, S.; Demont, P.; Lacabanne, C. Macromolecules 2000 33, 3425.

(29) Williams, G.; Watts, D. C. Trans. Faraday Soc. 1970, 66, 80.

(30) Williams, G.; Watts, D. C.; Dev, S. B.; North, A. M. Trans. Faraday Soc. 1971, 67, 1323.

(31) Vogel, H. Phys. Z. 1921, 22, 645

(32) Fulcher, G. A. J Am. Chem. Soc. 1925, 8, 339.

(33) Tamman, G.; Hesse, W. Z. Anorg. Allg. Chem. 1926, 156, 245.

(34) Angell, C. A. J . Non-Cryst. Solids 1991, 131-133, 13.

(35) Alves, N. M.; Mano, J . F.; Gómez Ribelles, J. L. J . Therm. Anal. Calorim. 2002, 70, 633.

(36) Alves, N. M.; Mano, J . F.; Gómez Ribelles, J . L. Polymer 2002, 43, 3627.

(37) McCrum, N. G.; Read, B. E.; Williams, G. Anelastic and Dielectric Effects in Polymeric Sol ids; Dover: New York, 1967.

(38) Mark, J. E. Rubber Chem. Technol. 1982, 55, 762.

(39) Trel oar, L. R. G. ThePhysics of Rubber Elasticity; Clarendon Press: Oxford, 1975.

(40) Mark, J . E., Ed. Physical Properties of Polymers Handbook; American Institute of Physics: New York, 1996.

(41) Ferry, J . D. Viscoel astic Properties of Polymers, 3rd ed.; J ohn Wiley \& Sons: New York, 1980.

(42) Williams, M. L.; Landel, R. F.; Ferry, J . D. J . Am. Ceram. Soc. 1953, 77, 3701.

(43) Ngai, K. L.; Rendell, R. W. J . J . Non-Cryst. Solids 1991, 131$133,942$.

(44) Roland, C. M.; Ngai, K. L. Macromolecules 1991, 24, 5315.

(45) Roland, C. M. Macromolecules 1992, 25, 7031.

(46) Pethrick, R. A.; Davis, W. J . Polymer 1998, 39, 255.

(47) Beiner, M. Macromol. Rapid Commun. 2001, 22, 869.

(48) Williams, G. Structure and Properties of Polymers. In Dielectric properties of Polymers in Materials Science and Technology Series; Thomas, E., Ed.; VCH: London, 1993; Vol. XII, p 471

(49) Gómez Ribelles, J . L.; Monleón Pradas, M.; Meseguer Dueñas, J. M.; Torregrosa Cabanilles, C. J . Non-Cryst. Solids 2003 307-310, 731

(50) Böhmer, R.; Ngai, K. L.; Angell, C. A.; Plazek, D. J . J . Chem. Phys. 1993, 99, 4201.

(51) Alves, N. M.; Mano, J . F.; Gómez Ribelles, J . L.; Gómez Tejedor, J . A. Polymer 2004, 45, 1007.

(52) Garwe, F.; Schönhals, A.; Lockwenz, H.; Beiner, M.; Schröter, K.; Donth, E. Macromolecules 1996, 29, 247.

(53) Williams, G. Trans. Faraday Soc. 1966, 62, 2091

(54) Alves, N. M.; Mano, J . F.; Gómez Ribelles, J. L. Macromol. Symp. 1999, 148, 437.

(55) Sauer, B. B.; Kim, Y. H. Macromolecules 1997, 30, 3323.

(56) Alegria, A.; Guerrica-Echevarria, E.; Telleria, I.; Colmenero, J. Phys. Rev. B 1993, 47, 14857-14865.

(57) Sauer, B. B.; Avakian, P. Polymer 1992, 33, 5128.

(58) Mudarra, M.; Belana, J .; Cañadas, J . C.; Diego, J . A. Polymer 1999, 40, 2659.

(59) Ediger, M. D.; Angel, C. A.; Nagel, S. R. J . Phys. Chem. 1996, $100,13200$.

(60) Plazek, D. J .; Ngai, K. L. Macromolecules 1991, 24, 1222.

(61) Roland, C. M.; Ngai, K. L. Macromolecules 1992, 25, 5765.

(62) Böhmer, R.; Ngai, K. L.; Angell, C. A.; Plazek, D. J . J . Chem. Phys. 1993, 99, 4201.

(63) Donth, E. J . Polym. Sci., Phys. Ed. 1996, 34, 2881.

(64) Huang, D.; McKenna, G. B. J . Chem. Phys. 2001, 114, 56215630.

(65) Ngai, K. L.; Roland, C. M. Macromolecules 1993, 26, 2688.

(66) Nielsen, L. E. Mechanical Properties of Polymers and Composites; Marcel Dekker: New York, 1974; Vol. 1.

(67) Saiter, A.; Devallencourt, C.; Saiter, J . M.; Grenet, J . Eur. Polym. J. 2001, 37, 1083.

(68) Colluci, D. M.; Mckenna, G. B.; Filliben, J J .; Lee, A.; Curliss, D. B.; Bowman, K. B.; Russel, J . D. J . Polym. Sci., Polym. Phys. Ed. 1997, 35, 1561.

(69) I to, K.; Moynihan, C. T.; Angell, C. A. Nature(London) 1999 $398,493$.

MA035626Z 